\section{TRIMming the response}

Viruses have evolved several mechanisms to evade or counteract the host antiviral immune response. Now, Gack et al. describe a new mechanism by which the influenza A virus nonstructural protein 1 (NS1) inhibits the production of type I interferons (IFNs) and antiviral immunity by preventing the ubiquitylation of the intracellular viral sensor retinoic acid-inducible gene I (RIG-I) by the ubiquitin ligase tripartite motif-containing 25 (TRIM25).

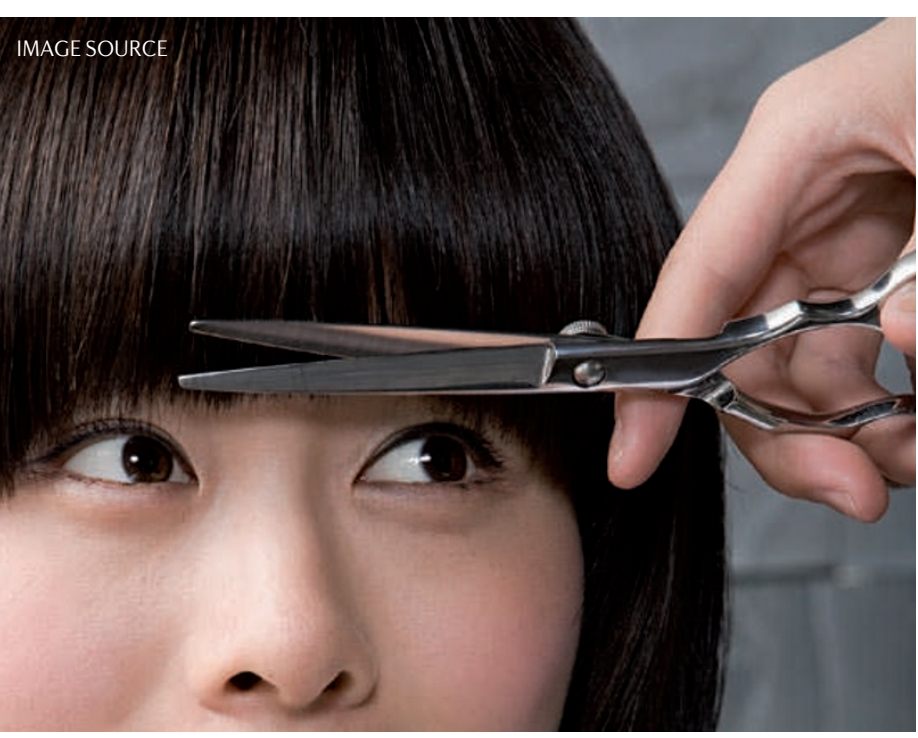

The recognition of viral RNA by RIG-I and the ubiquitylation of the second caspase recruitment domain (CARD) of RIG-I by TRIM25 initiates a downstream antiviral signalling cascade that induces the production of type I IFNs and other antiviral mediators. NS1 has previously been shown to antagonize RIG-I function and inhibit the production of type I IFNs, but the molecular mechanism involved was not known.

In this study the authors show that NS1 inhibits the ubiquitylation of RIG-I, which blocks the interaction of RIG-I with its downstream signalling partner MAVS (mitochondrial antiviral signalling protein). NS1 was shown not to target RIG-I directly but to interact with the coiled-coil domain of TRIM25, thereby preventing TRIM25 multimerization, which is required for the ubiquitylation of RIG-I. Further analysis showed that a Glu96Ala and Glu97Ala NS1 mutant could not bind and interfere with TRIM25 multimerization, inhibit the ubiquitylation of RIG-I or suppress IFN $\beta$ production.

But does targeting of RIG-I ubiquitylation by NS1 affect the pathogenicity of influenza A virus in vivo? Mice infected with wild-type virus succumbed to infection by 6 days after infection. However, mice that were infected with a mutant virus that expressed the Glu96Ala and Glu97Ala NS1 showed no signs of morbidity and had decreased pulmonary viral titres at 3 and 5 days after infection. In addition, the production of type I IFNs by mouse embryonic fibroblasts (MEFs) infected with the mutant virus was substantially higher than that of MEFs infected with the wild-type virus, and this higher type I IFN production was lost in MEFs from TRIM25-deficient mice.

So, the data show an important role for NS1-mediated inhibition of the host type I IFN response, achieved through the inhibition of TRIM25-mediated RIG-I ubiquitylation, in influenza A virus virulence and confirms that TRIM25 is an important host antiviral factor.

Olive Leavy

ORIGINAL RESEARCH PAPER Gack, M. U. et al. Influenza A virus NS1 targets the ubiquitin ligase TRIM25 to evade recognition by the host viral RNA sensor RIG-I. Cell Host Microbe 5, 439-449 (2009)

FURTHER READING Ozato, K., Shin, D.-M., Chang, T.-H. \& Morse, H. C. TRIM family proteins and their emerging roles in innate immunity. Nature Rev. Immunol. 8, 849-860 (2008) 